\title{
TEM Image Analysis of Self-Organized Large Gold Nanoparticle Arrays
}

\author{
Steven L. Tripp, Beomseok Kim, Alexander Wei
}

Department of Chemistry, Purdue University, West Lafayette, IN 47907-1393

The synthesis of nanostructured materials with tunable properties is central to developments in nanoscale science and technology. Self-assembly processes such as the spontaneous organization of monolayer-protected metal nanoparticles into periodic two-dimensional (2D) arrays are appealing due to their novel optical and electronic properties, which are a function of particle size or interparticle spacing [1]. Hydrophobic surfactants such as alkanethiols are often used to drive the nanoparticles toward self-organization at the aqueous interface; however, $2 \mathrm{D}$ array formation by this methodology has so far been successful only for small $(<10 \mathrm{~nm})$ metal particles [2,3]. Stabilized metal particles beyond this size threshold become increasingly prone to multilayer or threedimensional aggregate formation, which can be attributed to the rapid increase in van der Waals attraction and the loss of surfactant chain mobility on the planar facets of the nanoparticles as a function of size [4].

We have discovered that large gold nanoparticles $(16-170 \mathrm{~nm})$ encapsulated by resorcinarene tetrathiol 1 (Figure 1) are capable of self-organizing into 2D arrays. The arrays exhibit sizedependent extinction maxima, which shift toward near-infrared wavelengths with increasing periodicity. Careful inspection of the transmission electron microscopy (TEM) images reveals an inverse correlation between particle size within the array and interparticle spacing, an indication of increased van der Waals attraction with unit particle size. Periodicities obtained by particle density and fast Fourier transform analyses were not sufficiently precise to provide meaningful estimates for average interparticle separations, which ranged from 6 to less than $1 \%$ of the average particle diameter $(d)$. However, direct measurement of individual spacings within well-defined hcp domains enabled the calculation of spacing parameters $(\delta)$ with indeterminate errors defined by the digital resolution of the TEM images. These relative values clearly demonstrate a trend toward decreasing average interparticle spacings with increasing periodicity (Figure 2). Decreases in spacing are likely due to the greater van der Waals attraction between larger particles [5].

\section{References}

[1] Collier, C. P. et al., Annu. Rev. Phys. Chem. 49 (1998) 371.

[2] R. G. Osifchin, thesis, Purdue University (1994).

[3] To the best of our knowledge, the largest unit size reported for a close-packed 2D gold nanoparticle array is $18.5 \mathrm{~nm}$, prepared by electrophoretic deposition: Giersig, M.; Mulvaney, P. J. Phys. Chem. 97 (1993) 6334.

[4] Badia, A. et al., J. Am. Chem. Soc. 119 (1997) 2682.

[5] This research was supported by the National Science Foundation (BES-0086804) and the Research Corporation Innovation Award program (RI-0333). TEM images were taken at the Purdue Life Sciences Microscopy Facility. The authors thank Prof. David Thompson and Dr. Jong-Mok Kim (Purdue University) and Dr. Mary Beth Williams, Mr. Robert Johnson, and Prof. Joseph Hupp (Northwestern University) for use of their spectrophotometers and film deposition equipment and Prof. Alex King (Purdue University) for helpful discussions regarding TEM analysis. 

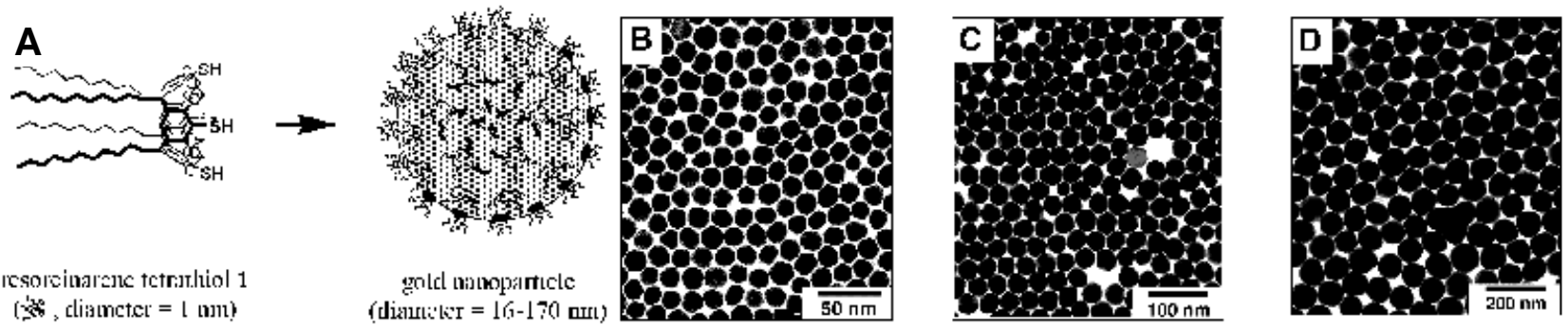

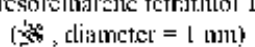

(dium:

Figure 1. Self-organized arrays of large gold nanoparticles. (A) Colloidal gold particles were first treated with resorcinarene tetrathiol $\mathbf{1}$, then dispersed at the air-water interface and transferred onto substrates. (B, C) TEM images of self-organized 2D arrays of encapsulated gold particles with average diameters of 16 and $34 \mathrm{~nm}$, respectively. Arrays were transferred onto Formvar-coated $\mathrm{Cu}$ grids (300 mesh) by manual Langmuir-Schaefer deposition. (D) TEM image of self-organized 2D array of encapsulated gold particles with an average diameter of $87 \mathrm{~nm}$. Array was transferred by mechanically controlled vertical deposition onto a Formvar-coated $\mathrm{Cu}$ grid mounted on a glass slide which had been ionized just prior to use.

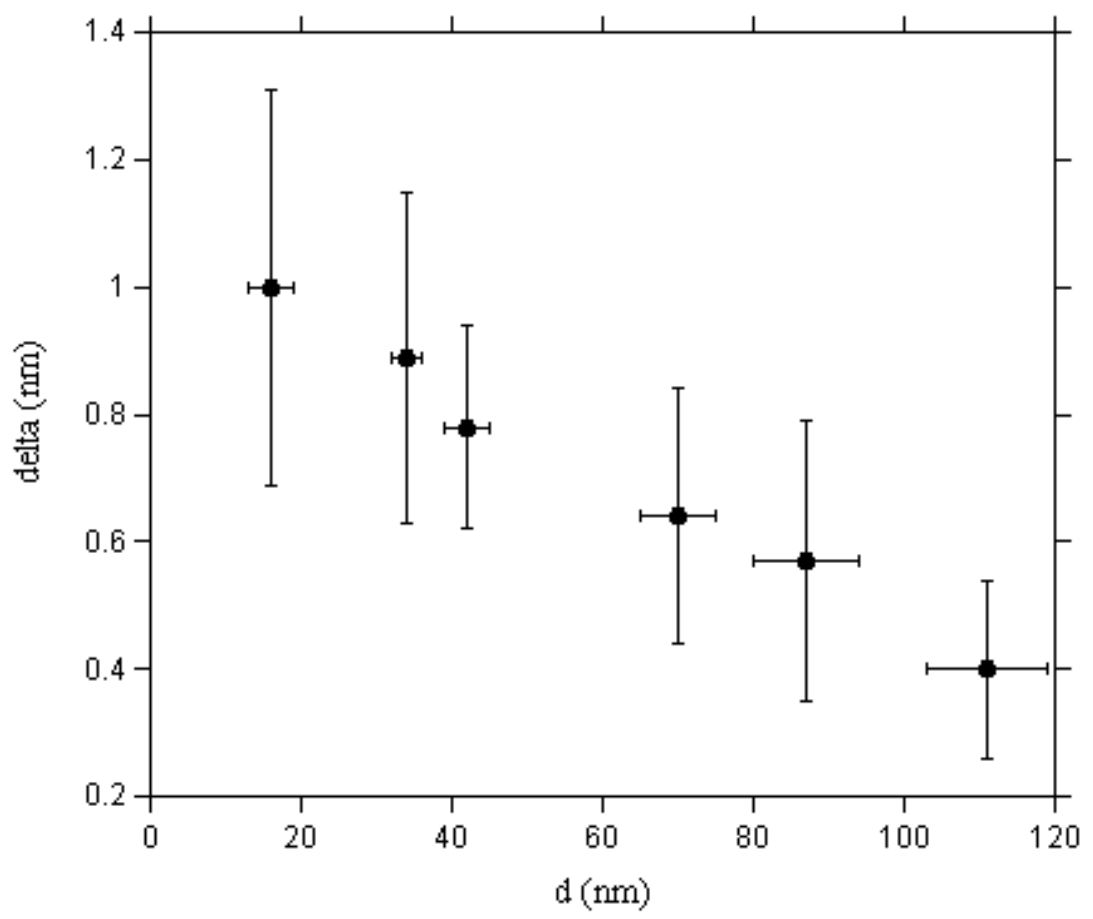

Figure 3. Average particle diameters $(d)$ versus interparticle spacing parameters $(d)$ of large gold nanoparticle arrays based on TEM image analysis.9 Errors are equal to one standard deviation from the mean. 